\title{
SPECULATIONS ON A STRONGLY INTERACTING HIGGS SECTOR
}

\author{
Martin B. EINHORN \\ Randall Laboratory of Physics, The University of Michigan, Ann Arbor, MI 48109, USA
}

Received 14 March 1984

\begin{abstract}
Using the $1 / N$ expansion, we argue that the $\mathrm{O}_{2 N}$ Higgs-Goldstone model may be a good indicator of the behavior of the standard $\mathrm{SU}_{2} \otimes \mathrm{U}_{1}$ electroweak model in the non-perturbative limit of a strongly interacting Higgs sector. We emphasize that there remains a physical scalar particle or resonance $\sigma$ (Higgs remnant), whose mass (and width) will be set by the weak scale. However, its coupling to vector bosons is expected to be much stronger than the standard model Higgs of comparable mass. This provides evidence that there is an upper limit to the Higgs mass in the hundreds of $\mathrm{GeV}$, regardless of whether naturalness constraints are imposed on the parameters of the effective lagrangian. We conclude with some comments about the possible relevance of this particle to the radiative events observed at the CERN $\bar{p} p$ collider.
\end{abstract}

In the standard electroweak model, the scalar Higgs field and its self-interactions are widely regarded as an effective field theory, valid on an energy scale below a few hundred $\mathrm{GeV}$ but, for reasons of naturalness, probably requiring significant modification above an energy scale of one or two TeV. The two most popular conjectures concerning the modification to be expected are low-energy supersymmetry (possibly softly broken) and composite models such as technicolor which regard the scalar Higgs field as a condensate of more fundamental strongly interacting fermions. Although supersymmetry retains the practical virtue of a reliable perturbative solution, there is as yet no experimental evidence for the plethora of super-partners and additional Higgs scalars which these models necessarily include. Technicolor theories, in addition to the difficulty of making predictions in a strongly interacting regime, tend to have other problems such as flavor-changing currents. An interesting question, regardless of the underlying theory, is what happens in the standard model as the Higgs mass becomes large [1-3]. Since the weak scale is fixed by the Fermi constant $G_{\mathrm{F}}$, this corresponds to increasing the scalar self-coupling $\lambda$. In perturbation theory, the Higgs mass is $m_{\mathrm{H}}^{2}=2 \mu^{2}=2 \lambda v^{2}$ where $v \cong 246 \mathrm{GeV}$. Perturbation theory breaks down for large $\lambda$, a guide being the violation of unitarity in the tree approximation which occurs for $m_{\mathrm{H}} \sim 1 \mathrm{TeV}$ [2]. This situation is frequently investigated by reference to the gauged, non-linear $\sigma$-model, which the standard model is presumed to approach in the limit $\lambda \rightarrow \infty$ for fixed $v^{2}=\mu^{2} / \lambda$. (We'll have more to say about this later.) 
If one is unwilling to fine-tune bare parameters, this limit seems inconsistent. For example, consider the quadratic divergence at one loop:

$$
-\mu^{2}=-\mu_{0}^{2}+\frac{\lambda \Lambda^{2}}{4 \pi^{2}}
$$

for a momentum cutoff $\Lambda$. The essence of naturalness [1,4] is expressed by the requirement that radiative corrections are not large compared to the physical mass. Consequently, $v^{2}=\mu^{2} / \lambda \geqslant \Lambda^{2} / 4 \pi^{2}$, so $\Lambda \leqslant 2 \pi v \approx 1.5 \mathrm{TeV}$. It clearly makes no sense to have $\mu_{0}$, the mass parameter of an effective local field theory, large compared to the momentum cutoff, so $\mu, \mu_{0} \leq 1.5 \mathrm{TeV}$, say. Consequently $\lambda=\mu^{2} / v^{2} \leq 4 \pi^{2}$. (In fact, the perturbative unitarity limit [2] corresponds to $\lambda \approx 8$.) Thus, the now conventional view is that the standard model makes no sense as an effective field theory beyond an energy scale of $1.5 \mathrm{TeV}$ and that the formal limit $\mu^{2}, \lambda \rightarrow \infty$ for fixed $v^{2}$ is to be interpreted in that context $\left(\mu \leq 1.5 \mathrm{TeV}, \lambda / 4 \pi^{2} \leqslant 1\right)^{\star}$.

A contrary point of view would be that in fact the origin of masses remains a big mystery, and so we do not yet know how much credence to attach to these naturalness concepts. If the standard model were substantially modified below 1.5 $\mathrm{TeV}$ or if certain relations obtained between fermion and boson masses which cancelled the quadratic dependence on the cutoff $\Lambda$ [1], as happens for example in supersymmetric models, then we could entertain the possibility that $4 \pi^{2} \mu^{2} / \lambda \ll \Lambda^{2}$, and, from this point of view, we can regard the cutoff $\Lambda$ and the mass parameter $\mu$ as independent parameters. Moreover, we shall further suppose that it is sensible to consider a strongly coupled limit of the effective scalar theory, however heretical this may seem in the conventional wisdom [5]. We shall entertain both the conventional and strong coupling points of view in the following.

Regardless of whether we believe the naturalness constraints $\left(\mu_{0} \leq \Lambda, \lambda_{0} / 4 \pi^{2} \leqslant 1\right)$ or whether we entertain the unconventional strong coupling limit $(\lambda \rightarrow \infty$ for fixed $\mu^{2} / \lambda$ ), if we want to consider $\lambda / 4 \pi^{2}$ near 1 , we must go beyond perturbation theory. Unfortunately, we do not have a reliable approximation scheme for the standard $\mathrm{SU}_{2} \otimes \mathrm{U}_{1}$ model. In the following, we consider instead a strongly interacting $\mathrm{SU}_{N} \otimes \mathrm{U}_{1}$ model. This has the advantage of admitting a non-perturbative but reliable solution to leading order in $1 / N$, but to all orders in the rescattering strength $\left(\lambda N / 4 \pi^{2}\right)$ so that the properties of a strongly interacting theory can be reliably inferred.

The standard $\mathrm{SU}_{2} \otimes \mathrm{U}_{1}$ model has an approximate global $\mathrm{O}_{4} \cong \mathrm{SU}_{2} \otimes \mathrm{SU}_{2}$ symmetry, which becomes exact in the limit of zero hypercharge $\left(\sin ^{2} \theta_{w}=0\right)$. After spontaneous symmetry breaking, there remains an approximate $\mathrm{O}_{3}$ global symmetry, which is responsible for the approximate equality of the W- and Z-masses. Suppose

* This sort of argument has been developed recently [5] but without reference to the quadratic dependence on $\Lambda$. 
we were to consider an $\mathrm{SU}_{N} \otimes \mathrm{U}_{1}$ theory instead of the standard model and took the complex scalar multiplet $\phi$ to be in the fundamental representation $N$ of $\mathrm{SU}_{N}$. Writing the scalar potential as

$$
V=\lambda\left(\phi^{+} \phi\right)^{2}-\mu^{2} \phi^{+} \phi
$$

we observe that it is $\mathrm{O}_{2 N}$ symmetric. To see this, let $\phi_{j} \equiv \sqrt{\frac{1}{2}}\left(\pi_{j}+i \pi_{N+j}\right)$, so that $\phi^{+} \phi=\frac{1}{2} \pi^{2}$, where $\pi$ is a $2 N$ component vector:

$$
V=\frac{1}{4} \lambda\left(\pi^{2}\right)^{2}-\frac{1}{2} \mu^{2} \pi^{2} .
$$

As usual, we take the ground state to have $\left\langle\pi_{j}\right\rangle=0, j \neq N$, and $\left\langle\pi_{N}\right\rangle=v$, with $v^{2}=\mu^{2} / \lambda$. Letting $\pi_{N}=v+\sigma$, the tree potential assumes the form

$$
V=\frac{1}{4} \lambda\left(\pi_{K}^{2}+\sigma^{2}\right)^{2}+\lambda v \sigma\left(\pi_{K}^{2}+\sigma^{2}\right)+\mu^{2} \sigma^{2},
$$

where $\pi_{K}^{2} \equiv \sum_{K \neq N} \pi_{K}^{2}$. Including gauge couplings, our strategy will be to analyze this theory in the limit $N \rightarrow \infty$ for fixed $\mu^{2}, \lambda N, g^{2} N, g^{\prime 2} N$, where $g\left(g^{\prime}\right)$ is the $\mathrm{SU}_{N}\left(\mathrm{U}_{1}\right)$ gauge coupling constant. The vector boson masses are $O(1)$ in this limit, for example $m_{\mathrm{V}}^{2} \equiv \frac{1}{4} g^{2} v^{2}=\mu^{2} g^{2} N / 4 \lambda N$. So long as the gauge couplings are small $\left(g^{2} N, g^{\prime 2} N \ll\right.$ $\lambda N$ ), the vector masses will be small compared to $\mu^{2}$. We shall usually be able to neglect the gauge couplings altogether. Note that the weak scale $v^{2}=\mu^{2} / \lambda$ is formally of order $N$.

This is generally thought of as a weak coupling limit since $\lambda$ is of order $N^{-1}$, however, the strength of rescattering corrections is of order $\left(\lambda N / 4 \pi^{2}\right)$ (see below) which is not necessarily small. Moreover, because of the occurrence of the factor of $1 / 4 \pi^{2}$, we can even entertain $\lambda>1$ so that the weak scale $v^{2}<\mu^{2}$. For example, a perfectly valid range of parameters is $1<\lambda N / 4 \pi^{2} \ll 2 N$ and $1 \ll \lambda \ll 8 \pi^{2}$. One approach to choosing parameters for purposes of comparison with the standard model is to set $m_{\mathrm{V}}^{2}=m_{\mathrm{W}}^{2}=(80 \mathrm{GeV})^{2}$ and $g^{2} N=2 g_{2}^{2}=0.85$, since these are held fixed as $N$ is increased. This determines $\mu^{2} / \lambda N=4 m_{\mathrm{V}}^{2} / \mathrm{g}^{2} N \approx(174 \mathrm{GeV})^{2}$. When a numerical illustration is desired, this is the value we shall use below. However, we do not expect to obtain reliable quantitative results for $N=2$ but hope to achieve qualitative insight into the non-perturbative, strong coupling regime.

Let us discuss the spectrum of theory. First, in the absence of vector fields and gauge couplings, we have an $\mathrm{O}_{2 N}$ symmetric theory spontaneously broken down to $\mathrm{O}_{2 N-1}$. As a result, there are $\frac{1}{2} 2 N(2 N-1)-\frac{1}{2}(2 N-1)(2 N-2)=2 N-1$ Goldstone bosons $\left(\pi_{K}\right.$ for $K \neq N$ ) and one massive scalar $\sigma$ (with tree mass $\sqrt{2 \mu}$ ). Suppose now we gauge the $\mathrm{SU}_{N}$ subgroup of $\mathrm{O}_{2 N}$. Of the $N^{2}-1$ vector fields, $(N-1)^{2}-1=N(N-2)$ remain massless (corresponding to the residual $\mathrm{SU}_{N-1}$ symmetry), while $2 N-1$ become massive, devouring all the would-be Goldstone bosons. (Note that none remain massless for $N=2$.) If we gauge-hypercharge as 
well, the initial $\mathrm{SU}_{N} \otimes \mathrm{U}_{1}^{Y}$ local symmetry is spontaneously broken down to $\mathrm{SU}_{N-1}$ $\otimes \mathbf{U}_{1}^{Q}$, so there is simply one more massless vector boson (photon).

Our primary interest is in the strongly interacting sector of the theory. At energies $E$ large compared to the vector boson masses (but not necessarily large compared to the Higgs boson mass), we may use the theorem [6] that the scattering amplitudes involving the longitudinal vector bosons and Higgs boson are identical (to order $m_{\mathrm{V}} / E$ ) to the scattering amplitudes in the broken symmetry phase of the scalars in the ungauged theory. Although the observations below are sufficiently interesting to warrant an investigation into the gauged theory itself, in this paper we will use this theorem to simplify the analysis and will treat the $\mathrm{O}_{2 N}$ scalar field theory. However, the reader should keep in mind that the amplitudes involving the Goldstone bosons ( $\pi_{K}$ for $K \neq N$ ) and massive boson ( $\sigma$ ) are to be thought of as the high-energy amplitudes for massive longitudinal vector bosons and the Higgs boson.

Assuming the gauge coupling constants are small, it is important to observe that, in the $\mathrm{SU}_{N} \otimes \mathrm{U}_{1}$ gauge theory, there is no strong interaction between those vector bosons which remain massless and either the Higgs boson or the other massive vectors. These interactions are determined by the strength of the gauge coupling constants.

If we can neglect the gauge couplings altogether, this $\mathrm{O}_{2 N}$ model is exactly solvable to leading order in $1 / N^{\star}$. The renormalized parameters are related to the bare parameters by

$$
\begin{gathered}
\frac{1}{\lambda N}=\frac{1}{\lambda_{0} N}+\frac{1}{4 \pi^{2}} \ln \frac{\Lambda}{M}, \\
-\frac{\mu^{2}}{\lambda N}=-\frac{\mu_{0}^{2}}{\lambda_{0} N}+\frac{\Lambda^{2}}{8 \pi^{2}},
\end{gathered}
$$

where $M$ is the normalization mass. Although these resemble the perturbative results, these are exact to leading order in $1 / N$ but to all orders in $\lambda N$. Note that the coupling $\lambda N$ is infrared-free and that, assuming $\lambda_{0} N>0$, there is an upper bound on the coupling strength at all scales $M<\Lambda$, given by

$$
\frac{\lambda N}{4 \pi^{2}}<\frac{1}{\ln (\Lambda / M)}
$$

Given some notion of the scale of the cutoff (say, $\Lambda \geqslant 1 \mathrm{TeV}$ ), the scale of strong rescattering corrections $\left(\lambda N / 4 \pi^{2} \geqslant 1\right)$ is very much restricted by this upper bound;

\footnotetext{
* This model has been analyzed in another context recently by Bardeen and Moshe [7], who were particularly concerned with the exact ground state. We want to emphasize that we treat this as an effective field theory with a bare mass $\mu_{0}$ and cutoff $\Lambda$, and we analyze the behavior at energies below $\Lambda$. Note also that their $N$ corresponds to our $2 N$. Other applications of the $1 / N$ expansion to this model in three dimensions have been discussed in [7a].
} 
it is apparently not possible to have strong rescattering at scales far below the cutoff. Since $\mu^{2} / \lambda N$ is scale invariant, $\mu^{2}$ decreases proportional to $\lambda N$ as the scale is reduced. Consequently, given $\mu^{2} / \lambda N$, eq. (7) translates into an upper limit on $\mu^{2}$ at scales below the cutoff:

$$
2 \mu^{2}=\frac{8 \pi^{2} \mu^{2}}{\lambda N} \frac{\lambda N}{4 \pi^{2}}<\frac{8 \pi^{2} \mu^{2}}{\lambda N} \frac{1}{\ln (\Lambda / M)} .
$$

Taking our previous value $\mu^{2} / \lambda N=(174 \mathrm{GeV})^{2}$, we have $2 \mu^{2}<(1.55 \mathrm{TeV})^{2}$ $(\ln (\Lambda / M))^{-1}$. This reinforces the conventional wisdom that the perturbative Higgs mass must be below the scale of the cutoff $\Lambda \approx 1.55 \mathrm{TeV}$.

What is the solution of the $\mathrm{O}_{2 N}$ scalar theory in the spontaneously broken phase? In leading order in $1 / N$, the propagator for the Goldstone bosons is unchanged, while the dominant contribution to the self-energy of the $\sigma$-field is given by the sum of bubbles depicted in fig. 1 . The result of this geometric series is

$$
\sum\left(p^{2}\right)=\frac{-4 \mu^{2} \lambda N I\left(p^{2}\right)}{1+2 \lambda N I\left(p^{2}\right)}
$$

where $I\left(p^{2}\right)$ is the standard bubble integral:

$$
\begin{aligned}
& I\left(p^{2}\right)=\int \frac{\mathrm{d}^{4} k}{(2 \pi)^{4}} \frac{1}{k^{2}(k+p)^{2}}+\text { counterterms, } \\
& I\left(p^{2}\right)=\frac{1}{16 \pi^{2}}\left|2+\ln \frac{M^{2}}{-p^{2}}\right|=\frac{1}{16 \pi^{2}} \ln \frac{e^{2} M^{2}}{-p^{2}} .
\end{aligned}
$$

Here $M$ is a normalization scale, and the logarithm is real for spacelike $p^{2}\left(p^{2}<0\right)$. Consequently, the inverse $\sigma$-propagator, $D_{\sigma \sigma}^{-1}=p^{2}-2 \mu^{2}-\Sigma\left(p^{2}\right)$, is

$$
D_{\sigma \sigma}=\frac{1+2 \lambda N I\left(p^{2}\right)}{p^{2}\left(1+2 \lambda N I\left(p^{2}\right)\right)-2 \mu^{2}} .
$$

The physical $\sigma$-particle occurs at the pole of $D_{\sigma \sigma}$. Of course, there is no pole for real $p^{2}$, since $I$ is complex for $p^{2}>0$ corresponding to the physical decay of the $\sigma$ into $\pi$ 's. To determine the position of the pole, we must solve for $s_{\sigma} \equiv\left(m_{\sigma}-\frac{1}{2} i \Gamma_{\sigma}\right)^{2}$ from

$$
s_{\sigma}=\frac{2 \mu^{2}}{1+\left(\lambda N / 8 \pi^{2}\right) \ln \left(e^{2} M^{2} /-s_{\sigma}\right)} .
$$

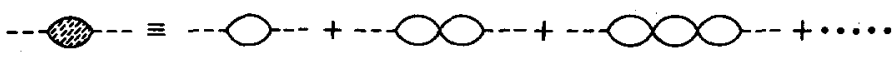

Fig. 1. The 1PI self-energy of the higgs. 
(Using eqs. (5) and (6), one can easily confirm that $s_{\sigma}$ is independent of the arbitrary scale $M$, as of course, a physical parameter must be.) Suppose we temporarily neglect the imaginary part (generally a poor approximation), and choose the normalization scale at $e M=m_{\sigma}$, then

$$
m_{\sigma}^{2}=2 \mu^{2}=\frac{8 \pi^{2} \mu^{2}}{\lambda N} \frac{\lambda N}{4 \pi^{2}}
$$

This simple equation has important consequences. Having found a scale on which the tree approximation equals the physical mass, we can use our previous upper limit (8) to deduce that

$$
m_{\sigma}^{2} \leqslant \frac{8 \pi^{2} \mu^{2}}{\lambda N} \frac{1}{\ln \left(e \Lambda / m_{\sigma}\right)}
$$

From the conventional approach [5], this lends support to the notion that the upper limit on the Higgs mass transcends perturbation theory. (Using the previous values for $\mu^{2} / \lambda N$, the bound is saturated for $m_{\sigma}=\Lambda=1.55 \mathrm{TeV}$.) From the strong coupling point of view, this equation is even more interesting. Trying to "freeze out" the Higgs particle by increasing the coupling constant $\lambda_{0} N$ indefinitely on scale $\Lambda$ (for fixed $\mu_{0}^{2} / \lambda_{0} N$ ), we find that there remains a pole in the Higgs propagator whose mass is given by eq. (12). We call this pole the Higgs remnant, although it continuously evolves from the perturbative Higgs particle. Except for unreasonably large values of the cutoff, the mass $m_{\sigma}$ of the Higgs remnant is set by $\mu^{2} / \lambda N$. Taking eq. (12) at face value, we see that it would be difficult for a strongly coupled theory to yield a mass scale much less than about $1 \mathrm{TeV}$. However, we must ask whether we believe the numerical conclusions from this $1 / N$ expansion for the real world at $N=2$. Of course, we cannot know the answer, but one question which gives some insight into this is how large must $N$ be for the $1 / N$ expansion to be numerically (rather than qualitatively) accurate. The actual value of the one-loop bubble graph with $2 N-1$ Goldstone bosons is proportional to $\lambda^{2}(2 N+7)$, so that the leading $N$ term is, for $N=2$, a factor of $\frac{4}{11}$ less than the actual value. The two-loop bubble is proportional to $\lambda^{3}\left(4 N^{2}+20 N+9\right)$, so that the leading $N$ contribution is, for $N=2$, actually only about one-fourth the actual value. (There is in addition another graph which increases the discrepancy.) This suggests that one cannot trust the numerical results for $N=2$ and that, if anything, the leading $N$ formula substantially underestimates the strength of the rescattering corrections. Consequently, the mass of the Higgs remnant could be much less than $1 \mathrm{TeV}$.

The inclusion of width effects is interesting and tends to lower the mass from the previous estimates. Let us return to the solution of eq. (12). It is desirable to choose $M$ to make it easy to solve for $s_{\sigma}$. To this end, we write $m_{\sigma}-\frac{1}{2} i \Gamma_{\sigma} \equiv e M R \exp (-i \theta)$ and choose the scale $M$ such that $R=1$, i.e. we normalize at $e M=\sqrt{m_{\sigma}^{2}+\frac{1}{4} \Gamma_{\sigma}^{2}}$. 
Then one finds that $M$ and $\theta$ are determined from

$$
\begin{gathered}
\tan 2 \theta=\frac{\lambda N}{8 \pi^{2}}(\pi+2 \theta) \\
e M=\sqrt{2 \mu^{2} \cos 2 \theta}
\end{gathered}
$$

Finally,

$$
m_{\sigma}=e M \cos \theta, \quad \Gamma_{\sigma}=2 m_{\sigma} \tan \theta .
$$

From eq. (15), we can see that the angle $\theta$ (and hence, $\Gamma_{\sigma} / m_{\sigma}$ ) is a monotonically increasing function of the coupling constant $\lambda N$. The mass can also be expressed as

$$
m_{\sigma}^{2}=\frac{8 \pi^{2} \mu^{2}}{\lambda N}\left[\frac{\sin 2 \theta(1+\cos 2 \theta)}{\pi+2 \theta}\right]
$$

Since $\mu^{2} / \lambda N$ is fixed and scale invariant, the variation of mass with coupling strength (or $\theta$ ) is determined by the quantity in brackets. The resulting values of $m_{\sigma}-\frac{1}{2} i \Gamma_{\sigma}$ are plotted in fig. $2 \mathrm{a}$, where we have also indicated the corresponding perturbative Higgs mass $m_{\mathrm{H}}=\sqrt{2 \mu}$. Of course, the latter is scale dependent, and we have rather arbitrarily chosen to compare to the perturbative value on a scale $M_{0}$ $\equiv \sqrt{m_{\sigma}^{2}+\frac{1}{4} \Gamma_{\sigma}^{2}}$. It is very striking that, once the coupling constant has increased

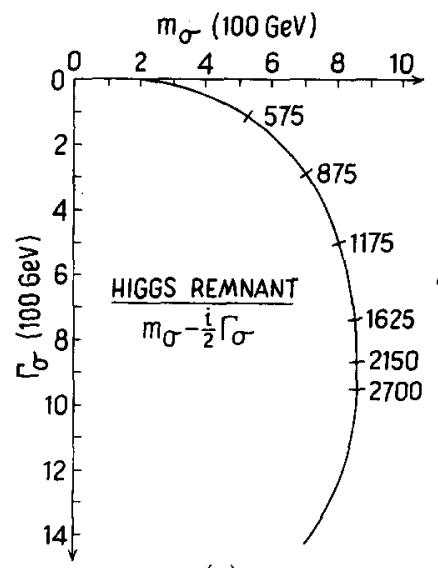

(a)

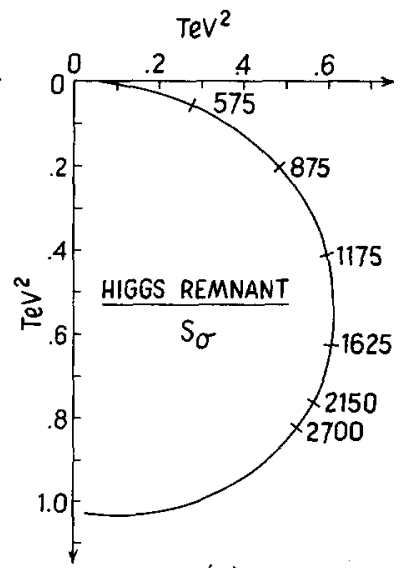

(b)

Fig. 2. (a) The position $m_{\sigma}-\frac{1}{2} i \Gamma_{\sigma}$ of the Higgs remnant as a function of the coupling strength. (b) The position of the pole $s_{\boldsymbol{\sigma}}$ of the Higgs remnant as a function of the coupling strength. The tick marks on the curves denote the corresponding value of the perturbative Higgs mass. (See text.) 
beyond the perturbative regime, the mass $m_{\sigma}$ rapidly saturates, even though the width continues to grow. Using the previous values, we find $m_{\sigma} \leqslant 870 \mathrm{GeV}$. In fact, the mass begins to diminish for even larger couplings, but this part of the curve is presumably not trustworthy because it corresponds to a regime in which $\lambda N \rightarrow \infty$ at a scale below $M_{\sigma}$. As another example, suppose $\lambda_{0} N=\infty$ at $\Lambda=1.55 \mathrm{TeV}$. Then we find $m_{\sigma}=869 \mathrm{GeV}, \Gamma_{\sigma} \approx 895 \mathrm{GeV}$. If we simply take these numbers at face value, the width of the Higgs remnant is roughly three times as large as the perturbatively calculated width for a standard model Higgs of the same mass.

For a large width, the traditional separation of $s_{\sigma}$ into $m_{\sigma}$ and $\Gamma_{\sigma}$ becomes meaningless; the only dynamically significant quantity is the position of the complex pole $s_{\sigma}$ itself, which is plotted in fig. 2b. (Since eq. (15) always admits a solution for $2 \theta<\frac{1}{2} \pi, \operatorname{Re} s_{\sigma}>0$.) With an imaginary part of the order of the real part, we cannot even think of the pole as an ordinary resonance, a pronounced peak in a cross section. Other ways to characterize the effects of the pole is to cite the energy $\sqrt{\operatorname{Re} s_{\sigma}}$ at which the s-wave phase shift in the elastic scattering amplitude (see eq. (19) below) passes through $\frac{1}{2} \pi$. In the preceding example $\left(s_{\sigma}=(869-i 448)^{2} \mathrm{GeV}^{2}\right)$, this occurs around $745 \mathrm{GeV}$, but the breadth is so great that even at $\sqrt{s}=100 \mathrm{GeV}$, the elastic amplitude (in pole approximation) has reached $\frac{2}{3}$ of its maximum value.

Taking into account our earlier observations about the strength of rescattering for $N=2$, it may turn out that the strong coupling upper limit on the mass $m_{\mathrm{o}}$ in the standard model would be well below the value estimated above from the $1 / N$ expansion. This would come about if the transition from weak to strong coupling came about rather abruptly, say, near the weak scale $v$, analogously to the rapid transition from strong to weak coupling (precocious scaling) around $1 \mathrm{GeV}$ in QCD. This suggests the possibility that, in the strong coupling limit of the standard model, the Higgs remnant will be a pole whose mass may be several hundred $\mathrm{GeV}$ but whose width is on the order of the mass. Consequently, the smaller the actual value of its mass, $m_{\sigma}$, the greater the ratio of its width to the perturbatively calculated width for a standard model Higgs of the same mass, $m_{\mathrm{H}}=m_{\sigma}$. Thus, the Higgs remnant may not appear phenomenologically to be a particle or resonance and may mistakenly be interpreted as a breakdown of the standard model rather than simply a manifestation of a strongly coupled standard model!

The picture of the Higgs remnant to which we have been led is a broad "resonance" in the hundreds of $\mathrm{GeV}$ range, strongly interacting with itself and with massive vector bosons, behaving generally like a Higgs boson with much enhanced couplings. As a check on our interpretation of the pole, we have calculated the wave function renormalization constant $Z_{\sigma}$, the residue of the pole in the $\sigma$-propagator (eq. (9)). We find simply

$$
Z_{\sigma}^{-1}=1-\frac{\lambda N s_{\sigma}}{16 \pi^{2} \mu^{2}}
$$


The second term can be written as

$$
\frac{\lambda N s_{\sigma}}{16 \pi^{2} \mu^{2}}=\frac{\sin 2 \theta \mathrm{e}^{-2 i \theta}}{2(\pi+2 \theta)},
$$

which yields a remarkably small change in $Z_{\sigma}(\leqslant 10 \%)$ regardless of the choice of parameters in the model.

The preceding calculations illustrate several points. (i) No matter how large the perturbative Higgs mass $\sqrt{2 \mu}$ or how large the coupling constant, there may remain a remnant of the Higgs, the $\sigma$-pole, in the hundreds of $\mathrm{GeV}$ range. This suggests that it would be a grave error to neglect this scalar degree of freedom. Note as before that the properties of the Higgs remnant are predominantly set by the vector mass and gauge coupling (weak scale). (ii) The effect of strong coupling has been to make the width much larger than a standard model Higgs of mass $m_{\sigma}$. As a result, the $\sigma$-particle may not even be observable directly as a resonance but only as a broad but strong enhancement in $J=0$ vector-boson interactions throughout the hundreds of $\mathrm{GeV}$ energy range. Nevertheless, its coupling is so strong that processes sensitive to longitudinal vector bosons may proceed at a much faster rate than in the standard model. (iii) There is only a single Higgs remnant, not a spectrum of excitations. We shall return to discuss points (ii) and (iii) again subsequently.

These observations evoke the speculation that the strong coupling limit of the standard model is not a non-linear $\sigma$-model, but an effective linear model in which the scalar field's self-coupling is essentially determined. Contrary to naive expectations, this strong coupling limit remains effectively renormalizable.

If it should turn out that the Higgs remnant has a mass below about $200 \mathrm{GeV}$, the preceding approximation to its width is invalid. In the $\mathrm{SU}_{N} \otimes \mathrm{U}_{1}$ gauge model, all the vector particles to which the $\sigma$ couples to leading order in $1 / N$ are massive, and so the neglect of the mass of the particle in the bubble diagram may grossly overestimate the $\sigma$ width. For example, for $m_{\sigma}<2 m_{\mathrm{V}}$, the particle would be stable (to $\mathrm{O}(1 / N)$ ). As a phenomenological estimate of the effect of vector mass, we simply give the particle in the bubble a mass $m_{\mathrm{V}}$ so that the bubble integral (eq. (10)) becomes instead

$$
I^{\prime}(s)=\frac{1}{16 \pi^{2}}\left\{2+\ln \frac{M^{2}}{m_{\mathrm{V}}^{2}}-\left(1-\frac{4 m_{\mathrm{V}}^{2}}{s}\right)^{1 / 2} \ln \left[\frac{1-4 m_{\mathrm{V}}^{2} / s+1}{1-4 m_{\mathrm{V}}^{2} / s-1}\right]^{1 / 2}\right\},
$$

or

$$
I^{\prime}=\frac{1}{16 \pi^{2}}\left\{2+\ln \frac{M^{2}}{m_{\mathrm{V}}^{2}}-2\left(\frac{4 m_{\mathrm{V}}^{2}}{s}-1\right)^{1 / 2} \tan ^{-1} \frac{1}{\left(4 m_{\mathrm{V}}^{2} / s-1\right)^{1 / 2}}\right\} \quad\left(\text { for } s<4 m_{\mathrm{V}}^{2}\right) .
$$

For $m_{\sigma} \approx 200 \mathrm{GeV}$, this will reduce its width to $\sqrt{1-\left(4 m_{\mathrm{V}}^{2} / m_{\sigma}^{2}\right)} \approx 60 \%$ of our 
previous estimate. Of course, for $m_{\sigma}<160 \mathrm{GeV}$, the Higgs remnant becomes nearly stable, decaying in $\mathrm{O}(1 / N)$ to two photons or $\mathrm{Z}_{0} \gamma$.

Veltman [1] has emphasized that there is no direct evidence for the Higgs particle of the (perturbative) standard model and, owing to the screening theorem, there are as yet no observables sensitive to a strongly interacting Higgs sector $(\lambda / 4 \pi \sim 1)$. He suggests that, while there may not be an elementary Higgs scalar, there may be bound states of massive vector bosons which would lead to apparent modifications of the standard model. He has recently suggested [8] that these bound states are responsible for the puzzling $\mathrm{e}^{-} \mathrm{e}^{+} \gamma$ and $\mu^{-} \mu^{+} \gamma$ events reportedly observed in the CERN $\bar{p}$ p collider [9] and possibly also like-sign dimuon events in neutrino scattering [10]. (In ref. [10] these events have been reviewed recently by Nash. Another recent paper with references to earlier literature is by A. Haatuft et al. The topic has also been reviewed in talks by Knoblock.) Lee, Quigg and Thacker [2] have shown that this strong interaction effect manifests itself dramatically as violations of perturbative unitarity in the $J=0$ isosinglet channel. They showed that, in tree approximation, this corresponds to an attractive force whose strength grows with energy and might lead to a low-mass bound state of vector bosons resembling the Higgs. However, a rough $N / D$ calculation, while apparently yielding a bound state, failed to confirm this hope.

It is interesting to compare our results with these aforementioned discussions. The $N / D$ calculation of ref. [2], by imposing elastic unitarity, is similar to the bubble sum of the leading approximation in the $1 / N$ expansion. (And, of course, unitarity will be satisfied order by order in $1 / N$.) However, the same rescattering corrections which tend to bind vector bosons shift the position of the pole in the $\sigma$-propagator. This suggests that our $\sigma$-particle and the one found by $N / D$ methods are in fact the same, especially when we note the striking similarity between our fig. $2 \mathrm{~b}$ and fig. 12 of ref. [2]. However, the o-particle seems to be not so much a bound state of vector bosons but rather the remnant of the Higgs particle, renormalized downward in mass due to strong interactions. This point of view is reinforced by the fact that we found only a single pole in the $\sigma$-propagator, whereas the bound state point of view suggests a spectrum of excitations. (Of course, if we started with more than one Higgs multiplet, we would anticipate more than one remnant.) Our picture is quite different from what is to be expected from the $1 / N$ expansion applied to QCD or from the "-onia" characteristic of the binding of heavy quarks. We believe the fundamental difference lies in the contrast between the asymptotically free coupling of QCD and the infrared free self-coupling $\lambda$ of the scalar field. The former permits a potential picture because the interaction is weak at short distance, whereas the latter looks more like an attractive short-range potential having only a single bound state. A central question is whether poles appear in other angular momentum channels or additional poles appear in the $J=0$ channel.

On the other hand, many of the physical effects which Veltman [8] conjectures for bound states would be shared by the Higgs remnant. These would include events 

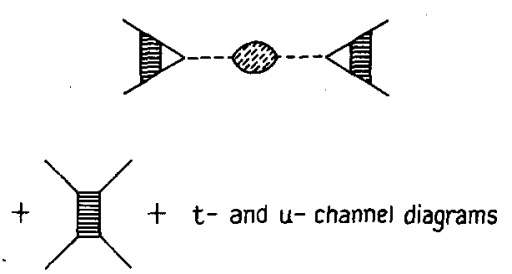

(a)

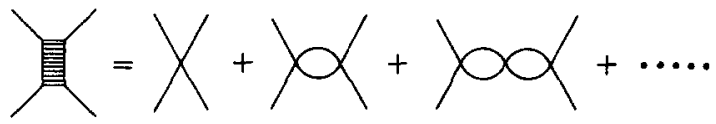

(0)

Fig. 3. (a) The elastic scattering amplitude consisting of one-particle reducible and irreducible diagrams.

(b) The leading diagrams contributing to the $s$-channel $1 \mathrm{PI}$.

with many jets beyond QCD background, jets accompanied by charged leptons and/or large missing energy, same-sign dileptons in neutrino scattering, and the copious production of weak vector bosons at very high energies. As we shall return to later, we would not expect substantial enhancement of radiative channels.

To clarify this distinction from a bound state picture further, let us discuss the elastic scattering amplitude for the Goldstone bosons (longitudinal vectors) to leading order in $1 / N$ (fig. 3). There are two contributions: the one-particle reducible (1PR) contributions involving the $\sigma$-propagator and the one-particle irreducible (1PI) diagrams contributing to $\Gamma^{(4)}$. The final result [8] is

$$
T=\frac{-2 i \lambda s \delta_{i j} \delta_{k l}}{s[1+2 \lambda N I(s)]-2 \mu^{2}}+t \text { - and } u \text {-channels }
$$

Once again we see the pole coming from the $\sigma$-propagator. But the $1 \mathrm{PI}$ rescattering corrections (fig. $3 \mathrm{~b}$ ) do not contribute to the pole. It is in this sense that we argue that the $\sigma$-pole is not truly a bound state but rather the remnant of the Higgs. (Of course, the driving force for the $N / D$ calculation of ref. [2] involves both 1PR and 1PI diagrams, so it is conceivable that they found the same effect.)

We remarked earlier that, for strong coupling, the $\sigma$-width was much greater than in the perturbative theory. For general purposes of comparison, it is useful to have a formula for the coupling $G_{\sigma \pi \pi}$ of $\sigma$ to the Goldstone bosons. This can be obtained from the width

$$
(2 N-1) G_{\sigma \pi \pi}^{2} \equiv 32 \pi m_{\sigma} \Gamma_{\sigma},
$$

where $2 N-1$ represents the number of decay channels. One can easily check that $G_{\sigma \pi \pi}$ agrees with the usual expression $(2 \sqrt{\lambda \mu})$ for weak coupling. In the strong 
coupling limit discussed earlier, we found that $\Gamma_{\sigma} \sim m_{\sigma}$, so we estimate

$$
\sqrt{2 N-1} G_{\sigma \pi \pi} \approx 4 \sqrt{2 \pi} m_{\sigma} \approx 16 \pi \sqrt{\mu^{2} / \lambda N} .
$$

Notice again that the lighter the mass $m_{\sigma}$, the greater the discrepancy between the perturbative calculation and the value in the strong coupling limit.

If, perchance, the $\sigma$-particle mass were below threshold for decay into massive vector bosons $\left(m_{\sigma}<2 m_{\mathrm{V}}\right)$, the preceding calculation is invalid. The coupling constant $G_{\sigma \pi \pi}$ must be inferred from the residue of the pole in the elastic scattering amplitude, eq. (21), where for the bubble integral we must use eq. (20b). Thus $m_{\mathrm{a}}^{2}$ is the solution of

$$
m_{\sigma}^{2}=\frac{2 \mu^{2}}{1+2 \lambda N I^{\prime}\left(m_{\sigma}^{2}\right)}
$$

It is convenient to choose the normalization scale $M$ where $I^{\prime}=0$, that is, letting $z \equiv \sqrt{\left(4 m_{\mathrm{V}}^{2} / m_{\mathrm{\sigma}}^{2}-1\right.}$

$$
\ln \frac{e M}{m_{\mathrm{V}}} \equiv z \tan ^{-1} \frac{1}{z}
$$

Then we find from the residue at the pole in $T$,

$$
2 N G_{\sigma \pi \pi}^{2}=\frac{16 \pi^{2} m_{\mathrm{o}}^{2}}{4 \pi^{2} / \lambda N+8 \pi^{2} m_{\mathrm{o}}^{2} \partial I^{\prime} / \partial s},
$$

where a simple calculation gives

$$
8 \pi^{2} m_{\sigma}^{2} \frac{\partial I^{\prime}}{\partial s}=\frac{1}{2 z}\left[\left(1+z^{2}\right) \tan ^{-1} \frac{1}{z}-1\right] .
$$

This coupling strength can be compared with the perturbative value $2 N G_{\sigma \pi \pi}^{2}=$ $4 N \lambda\left(2 \mu^{2}\right)$. By comparison, it is as if the coupling constant were substantially increased without increasing the mass. Thus, we anticipate a relatively large effective coupling strength between the Higgs remnant and massive vector bosons.

In summary, we have suggested that, via the $1 / N$ expansion, the $\mathrm{O}_{2 N}$ model can provide a reliable guide to the strong coupling limit of the $\mathrm{SU}_{N} \otimes \mathrm{U}_{1}$ model. It is our hope that it is also a reasonable qualitative description for the standard model $(N=2)$ for a strongly interacting Higgs sector. We found that the effects of rescattering can renormalize the Higgs mass down from the multi-TeV range to the hundreds of $\mathrm{GeV}$ range, and suggest that, contrary to naive expectations based on the non-linear $\sigma$-model, a strong interacting Higgs sector always leaves a scalar remnant. Regardless of whether one adopts the naturalness constraints or the strong coupling point of view, we have provided substantial evidence for a non-perturbative upper limit on the physical mass and width.

The distinction between an elementary and a composite state is semantic in a strongly interacting theory, and the $\sigma$-pole can equally be regarded as the Higgs or 
as a composite state of two vector bosons. The fact that we found only one such state together with the modest wave function renormalization (eq. (19)) lends itself to the elementary interpretation. On the other hand, the $N / D$ calculation of ref. [2] suggests that a sufficiently strong attractive force between massive vector bosons would regenerate the same result. The message seems to be that an effective scalar degree of freedom will always accompany a consistent theory of massive vector bosons.

The implications of this picture for the anomalous CERN events are presently unclear. Naturally, we wish to entertain the possibility that this Higgs remnant is being produced in the CERN p $\overline{\mathrm{p}}$ collider and may be'somehow responsible for the "non-standard" observations made by experiments UA1 and UA2. It is perhaps worth noting again that, if $m_{\mathrm{o}}<2 m_{\mathrm{W}}$, it is stable to leading order in $1 / N$. If $m_{\sigma}<m_{Z_{0}}$, the dominant decay will be to two photons in next order. Because $\left(N G_{\sigma \pi \pi}^{2}\right)$ is so much larger than in the standard model, this rate could be much faster than anticipated. If, however, $m_{\sigma}>m_{Z_{0}}$, it can decay in order $1 / N$ to $Z_{0} \gamma$ and, in the absence of fermions, this would be the dominant decay mode! (The same is true of the standard Higgs model.) However, even if the $\sigma$ does not couple directly to fermions, it is hard to understand why the $Z_{0} \gamma$ branching ratio would compete with $\mathrm{W}+2$ jets or with 4 jets. The latter may not be so much different from $Z_{0} \gamma$ because of phase space factors but the former appears to dominate by far. What is needed to understand the CERN observations is for the enhancement discovered here not to contribute, for example, to $W+2$ jets, but this requires a careful sorting out of the longitudinal and transverse modes of the gauge theory, a subject to which we intend to return. At present, it remains a mystery why radiative decays would be so prevalent.

I wish to thank P. Bracken and G.L. Kane for discussions of the properties of the standard model Higgs and M. Duncan, R. Phillipe, M. Veltman, and Y.-P. Yao for conversations concerning the strongly coupled Higgs sector and non-linear $\sigma$-model.

\section{Note added in proof}

Since this paper was written, "anomalous" events in non-radiative channels have been observed at the CERN SppS. See UA1 collaboration, Phys. Lett. 139B (1984) 115 and UA2 collaboration, Phys. Lett. 139B (1984) 105.

\section{References}

[1] M. Veltman, Acta Phys. Polonica B8 (1977) 475

[2] B.W. Lee, C. Quigg and H.B. Thacker, Phys. Rev. D16 (1977) 1519

[3] T. Appelquist and C. Bernard, Phys. Rev. D22 (1980) 200;

A. Longhitano, Phys. Rev. D22 (1980) 1166;

T.A. Appelquist, Lectures at the 21st Scottish Universities Summer School in Phys., St. Andrews, 1980 
[4] G. ' $t$ Hooft, Lecture II in recent developments in gauge theories, Proc. NATO ASI, Cargèse, France, Aug. 26-Sept. 8 1979, ed. G. 't Hooft, C. Itzykson, A. Jaffe, H. Lehmann, P.K. Mitter, I.M. Singer and R. Stora (Plenum, New York; 1980)

[5] R. Dashen and H. Neuberger, Phys. Rev. Lett. 50 (1983) 1897;

M.A. Beg, C. Panagiotakopoulos and A. Sirlin, Phys. Rev. Lett. 52 (1984) 883;

D.J. Calloway, Nucl. Phys. B233 (1984) 189

[6] J.M. Cornwall, D.N. Levin and T. Tiktopoulos, Phys. Rev. D10 (1974) 1145; Ref. [2], appendix

[7] W.A. Bardeen and M. Moshe, Phys. Rev. D28 (1983) 1372

[7a] T. Appelquist and R.D. Pisarski, Phys. Rev. D23 (1981) 2105;

T. Appelquist and U. Heinz, Phys. Rev. D24 (1981) 2169; D25 (1982) 2620

[8] M. Veltman, Phys. Lett. 139B (1984) 307

[9] UA1 Collaboration, G. Arnison et al., Phys. Lett. 122B (1983) 103;

UA2 Collaboration, M. Bannèr et al., ibid. 122B (1983) 476;

UAl Collaboration, G. Arnison et al., ibid. 126B (1983) 398;

UA2 Collaboration, P. Bagnaia et al., ibid. 129B (1983) 130;

UAl Collaboration, G. Arnison et al., ibid. 129B (1983) 273

[10] T. Nash, in Proc. 1983 Int. Symp. on Lepton and photon interactions at high energies, ed. D.G. Cassel and D.L. Kreinick, Cornell University, Ithaca, 1983, p. 359;

A. Haatuft et al., Nucl. Phys. B222 (1983) 365;

J. Knoblock, J. Allaby, M. Willutzky and T.Y. Lin, in Neutrino 81 Proc., vol. 1, University of Hawaii, Honolulu, 1981, pp. 421-466 